\title{
Correction to: Evaluating semantic relations in neural word embeddings with biomedical and general domain knowledge bases
}

Zhiwei Chen ${ }^{1 \dagger}$, Zhe He${ }^{2^{*}+}$, Xiuwen Liu ${ }^{1}$ and Jiang Bian ${ }^{3}$

\section{Correction}

After publication of this supplement article [1], it was brought to our attention that the Results section of the abstract contained a partial sentence. The partial sentence is as follows:

"Regarding the retrieval of semantic relations, we were able to retrieve semanti."

This sentence should be:

"Regarding the retrieval of semantic relations, we were able to retrieve diverse semantic relations in the nearest neighbors of a given word."

\footnotetext{
Author details

'Department of Computer Science, Florida State University, Tallahassee, FL, USA. ${ }^{2}$ School of Information, Florida State University, 142 Collegiate Loop, Tallahassee, FL 32306, USA. ${ }^{3}$ Department of Health Outcomes and Biomedical Informatics, University of Florida, Gainesville, FL, USA.
}

Received: 10 August 2018 Accepted: 15 August 2018 Published online: 22 August 2018

\section{Reference}

1. Chen Z, He Z, Liu X, Bian J. Evaluating semantic relations in neural word embeddings with biomedical and general domain knowledge bases. BMC Med Inform Decis Mak. 2018;18(Suppl 2):65. https://doi.org/10.1186/s12911-018-0630-x.

\footnotetext{
* Correspondence: zhe.he@cci.fsu.edu

${ }^{\dagger}$ Zhiwei Chen and Zhe He contributed equally to this work.

${ }^{2}$ School of Information, Florida State University, 142 Collegiate Loop,

Tallahassee, FL 32306, USA

Full list of author information is available at the end of the article
} 